\title{
The EnLighTable: Design of Affordances to Support Collaborative Creativity
}

\author{
Lucia Terrenghi ${ }^{1}$, Torsten Fritsche ${ }^{2}$, Andreas Butz ${ }^{1}$ \\ University of Munich, Media Informatics \\ Amalienstrasse 17, 80333 Munich, Germany \\ ${ }^{1}\left\{\right.$ lucia.terrenghi; butz\}@ifi.lmu.de; ${ }^{2}$ fritsche@cip.ifi.lmu.de
}

\begin{abstract}
In this paper we discuss some interface design concepts for supporting and enhancing collaborative creativity in multi-user interactive environments. We focus on the design of affordances for direct manipulation and collaboration on table-top displays. As an application of our concepts, we introduce the EnLighTable, an appliance for creative teamwork in the selection of pictures and layout design based on a table-top touch-sensitive display. We present our rationale for the design of affordances which metaphorically relate to artifacts - and map to gestures - of the physical world. Finally we discuss the results of our first design iteration and introduce a possible future extension of the table-top appliance to other devices of an instrumented environment.
\end{abstract}

\section{Introduction}

In the vision of ubiquitous computing, the walls and surfaces of our everyday life environments might have computing, display, and interaction capabilities [26]. This will change our mental model of a computer, which is currently mostly associated with a desktop workstation. Computers in the everyday life won't necessarily be Personal Computers: their appearance, on a physical as well as on a digital level, will need to support novel conceptual models concerning what a computer is, and how to work with it, individually and/or cooperatively.

The embodiment [8] of interaction in our physical as well as our social settings will create novel, more natural and casual contexts of interaction, in which people will deal with digital information in a more immediate way. Shared displays can support such a transition from personal to social interaction technically. On a conceptual level, we need visualization and interaction techniques which effectively support users' interaction among each other and with the machine. Novel contexts of colocated collaboration might also alter traditional communication and work-flows, thus affecting collaborative creativity.

In this paper we describe the EnLighTable. It is an appliance based on a table-top touch-sensitive display for creative teamwork in the selection of pictures and layout design, e.g. in advertising agencies. It enables multiple users to simultaneously manipulate digital pictures of a shared collection, and rapidly create and edit simple page layouts. In section 2 we consider the table as an information display and look at the 
work on interaction techniques that has been done in this field. In section 3 we consider the design of affordances for multi-user environments and the main issues to take into account in our scenario. In chapter 4 we introduce the EnLighTable appliance and motivate our design goals. We then present our design solutions as we report on our design of affordances for multi-user direct manipulation of digital information on a table-top display. Finally, we present the results of the first design iteration of the project and discuss future work.

\section{The Table as an Information Display, Related Work}

Tables are used for many daily activities in societies of most cultures. Coffee tables, desktops, meeting tables support different social as well as individual activities, leisure or work related. Tables can easily be shared by several people, who then comfortably face each other with the table between them. This allows easy verbal and gesture-supported face to face communication with the table surface permanently in sight. Tables create a shared space for the collaborative manipulation of objects. Scott et al. found [20] that humans tend to divide this space into different functional areas. In particular, the middle of a table is often used as a shared area for the exchange of objects, while areas closer to a specific user are considered increasingly private by this user. When dealing with rotation-dependent objects, such as text or pictures, users tend to reorient them for their own viewing direction, which can cause conflicts of interest with other users. On the other hand, when a user reorients an object towards another person, this action shows her intention to discuss the object with this person.

Since Wellner's digital desk [27], Krüger's responsive workbench [17] and Ullmer's Metadesk [25], research has investigated various forms of interaction with table-tops for a wide variety of tasks [18], [22], [19]. Closest to our own work are Hinrichs' Interface currents [14] and Apted's SharePic [1] which both deal with the task of collaborative picture selection.

Interface Currents are circular streams in which the images float around the table. As they pass each user, they face her in the right orientation, which nicely solves the orientation problem. Pictures can be fished from the stream and dragged to a private bank between the stream and the table's border. While this metaphor does provide affordances for the manipulation of the images, it is a general purpose metaphor and as such rather unrelated to the physical manipulation of photos. Furthermore, it does not support spatial memory and the possibility to share a consistent visual landscape among team members, because the pictures continuously flow in the currents.

SharePic emphasizes the different functional areas of the table, but leaves the orientation problem to the user. Pictures can be freely rotated, scaled and annotated, but no automatic reorientation is done. While this behavior is closer to the physical world, in which photos have to be rotated by the user as well, it doesn't use the additional capabilities of the new medium in this respect. 


\section{Affordances for Multi-User Interactive Environments}

In Terrenghi [24] we presented our work on design of affordances for direct manipulation of digital information. We introduced an interaction paradigm in which surfaces act as interfaces and hands as control devices. Thus, we discussed the design of graphical user interfaces supporting hands-on direct manipulation of digital information in the environment. In order to support users' gesture-based interaction, we suggested the use of affordances. Their design can either rely on the visual appearance of the information, or on the metaphoric link to real world objects and their affordances in the physical world.

The work we describe in this paper builds on those insights and extends the design space to multi-user interaction on a large horizontal display. Specifically, we look at the manipulation of digital picture collections in co-located collaboration. The collaborative setting adds some complexity to the design of affordances, such as:

- Physical arrangement of multiple users around the table;

- Issues of territoriality, reachability, sharability and passing on;

- Orientation of information.

We distinguish between physical and cognitive affordances, as suggested in [13]. Physical affordances are design features which help users to execute a physical action in the interface. Some physical affordances are provided by the table itself, as an artifact: It allows several users to gather around it; it allows the display of information and at the same time supports physical objects; it naturally fosters two-handed interaction by supporting both forearms. Cognitive affordances, on the contrary, are design features which tell users something, mostly about a physical action they can do with the interface. To give an example from the physical world, a cognitive affordance could be the beer mat we put on the table. It offers a visual indication of where to place the beer glass so as to avoid drops on the table. The visibility of the beer mat and its physical placement on the table tell users what to do with the glass on a cognitive level. Most cognitive affordances are based on conventions, and there is nothing intrinsic in the appearance of the artifact that suggests what it is for.

On top of the distinction between cognitive and physical affordances, social affordances have been defined [16]. These are properties of collaborative environments which serve as facilitators of the social context. Thy enable and provoke social interaction between a member and the group (i.e., perception and action coupling). Perception and action are then the results of the intentions of the group member and of the social affordances of the collaborative environment. As an example in the physical world, a couch provides social affordances for people to sit next to each other. Gaver [10] explores an ecological approach to social interaction using the concept of affordances to describe material properties of the environment that affect how people interact. From his perspective, the design of new technologies can rely on existing affordances provided by the physical environment to elucidate and analyze human behaviors in a new light.

This means that our design needs to consider the following aspects: 
- existing physical, as well as social affordances provided by the table as a physical artifact,

- design of cognitive affordances for the manipulation of digital information,

- design of social affordances for collaboration.

This implies an approach to the design and study of hybrid (i.e., physical and digital) technological artifacts that considers their social context of use. In Grint and Woolgar [11] "Technologies, do not 'by themselves' tell us what they are or what they are capable of. Instead, capabilities - what, for example, a machine will do - are attributed to the machine by humans. Our knowledge of technology is in this sense essentially social”.

In the next section we describe the EnLighTable, a multi-user table-top display appliance. In its design, we tried to merge the principles above, in order to create an interactive environment which supports collaborative creativity.

\section{The EnLighTable Appliance}

The EnLighTable is an appliance which aims to support picture selection and layout design in creative teamwork, e.g. in advertising agencies, publishing companies, or catalogue production companies. The cheap production of digital pictures causes a dramatic growth of picture collections. Creative directors, art directors, and graphic designers often need to select the pictures for a defined communication project from a large collection. Selecting the right picture for a flyer, a catalogue or an advertising campaign usually involves several individuals in the discussion, in iterative phases of communication. The patterns of communication may change according to the size of the company and of the project, the hierarchical organization of the company, or the source of the picture collection (cf. Section 6.1). Often, a strategic concept for the communication is initially defined together in a team, and then the different professionals "migrate" to their workstations to work on their individual sub-tasks. Afterwards, they meet again with print-outs of ideas, or move to each other's PCs to visualize and discuss ideas. Most of the creative phase of selecting pictures happens in parallel, in different locations, with limited communication.

Our design aims to explore the potential of digital table-top displays to support collaborative creativity in co-located work. In Fischer [9] Creativity occurs in the relationship between an individual and a society, and between an individual and his or her technical environment. Appropriate socio-technical settings, at the same time, can amplify the outcome of a group of creative people by both augmenting individual creativities and multiplying rather than simply summing up individual creativities. From this perspective the physical, social and interactive contexts acquire a main role in determining cognitive processes. In [23] collaborative creativity is described as a social and communicative transaction between people who in some way share a mutual goal. In this sense an act which is objectively creative is not necessarily "collaboratively creative" if it does not support a shared goal or is not recognized as a contribution by the other team members. 
The layout of the environment plays a major role in the perception of ideas [22][23]. Furthermore, visibility of action is a main design principle for embodied interaction [8]. It provides awareness of what other colleagues are doing and how the actions of group members affect the shared artifacts. This relies on existing theories from CSCW [6] [7]. Group awareness (i.e. the condition where members perceive the presence of other group members and the possibility to communicate with them), seems to provide chances for informal communication. Informal communication facilitates the transfer of essential information related to task-specific activities. Most interactions in the work environment take place during chance encounter.

In the EnLighTable appliance we expect the mutual visibility and awareness of others' interactions to provide affordances for discussion and collaborative creativity in the early stage of layout-design. Therefore the design of the appliance on our tabletop display focused on the following requirements:

- Up to 3 users can simultaneously see and reach a shared picture collection: each user can see what others select and drag objects out of the collection

- Each user can pick some pictures out of such a collection, without affecting the others' range of selection: thus, each user works on copies of picture items, while the originals are maintained in the shared collection

- Each user can operate basic manipulation tasks on the selected pictures, and save the changes as edited instances

- Each user can draft page layouts on the table and place pictures within the layout frames.

In the following section we describe how the design of the graphical user interface tries to meet the requirements above.

\section{Design of Affordances: Borrowing from the Physical World}

The graphical user interface for direct manipulation of information items by multiple users was designed on three different levels:

- design of information arrangement for social interaction

- design of information items for direct manipulation

- design of a virtual tool for additional editing and manipulation of information items.

The three levels are discussed separately in the following.

\subsection{Information Arrangement for Social Interaction}

The arrangement of information items on the table needed to cope with the constraints and affordances of the horizontal surface. When users sit at the sides of a table, personal areas of interaction are implicitly determined by people's area of reach 
and visual angle, as in the normal physical space. Metaphorically, we adopted an arrangement similar to the one of a set table (see fig. 1, (a)). The perception of a personal area of interaction is suggested by the pre-defined placement of three Imagetools (cf. section 5.3) oriented towards the sides of the table. In analogy to plates, this is expected to suggest "guests" where to sit, i.e., their personal area of interaction. In the center, a bigger shared container of information is displayed. It contains the thumbnails of a shared picture collection, e.g. the pictures of a photo shooting or of a shared library. The tray can be dragged with a simple gesture towards the personal area of interaction (see fig. 1, (b)). When such a "shared tray" is dragged in the direction of one of the Imagetools, the pictures reorient towards the dragging user. In this way, she can have a better view of the pictures, she can scroll to see more, and drag the preferred ones out of it: i.e., she can "serve" herself with the desired information items. After the user ceases interacting with the shared tray, the latter automatically returns to the original orientation and location. In this way, a consistent spatial arrangement of the pictures is mostly maintained, thus supporting spatial memory for every user. On the other hand, the personal reorientation of the picture collection is allowed, which is important for visualizing and selecting pictures in the design phase. In the same tray, which plays the role of a shared resource, a bin is available. In the same interaction manner, users can move the shared tray towards them and drag the copies which are lying on the table to the bin in order to delete them.

(a)
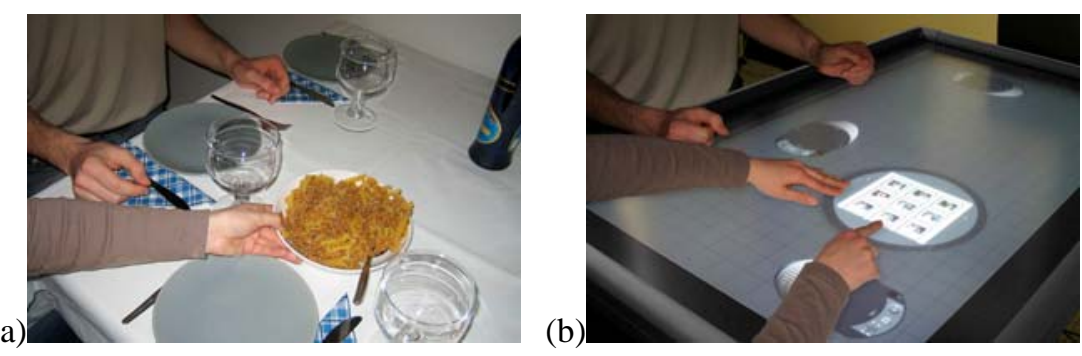

Fig. 1. Affordances for social interaction on the table. (a) Arrangement of physical artifacts in the everyday life. (b) Arrangement of digital information items on the EnLighTable appliance.

\subsection{Information Items for Direct Manipulation}

In the real world, negatives or slides are the original sources for analogue photography. In design and photographic working scenarios, slide film is often used and slides are projected, printed, scanned, i.e., edited and processed. Furthermore, they are often arranged on light tables, so as to visualize and compare multiple pictures simultaneously (see fig. 2, (a)). The cognitive relevance of humans' direct manipulation and spatial arrangement of artifacts have been studied by Kirsch [15]. In his study on people's intelligent use of space, Kirsch classifies spatial arrangement that simplify choice, perception and internal computation. In this sense, external representation and 
spatial arrangement support creativity as they enable the visualization of different possible solutions.

The white plastic frames of physical slides afford their manipulation, so as to handle them without ruining or occluding the negative. In analogy to this conceptual model, our information items visually resemble slides (see fig. 2, (b)). The size of our virtual slides ( $75 \times 75$ pixels) is such that their picture content can easily be recognized at a glance. When displayed in the shared tray, the slide frames are white, as their physical counterparts usually are. In the white area the copyright or source of the picture can be displayed, just as the physical slide frame can be labeled with a pen. Consistently with this metaphor, our digital slides represent the original source of information and provide affordances for manipulation and editing. When users touch the original slide on the shared tray and drag it to empty areas of the table surface, a copy of the slide is created. The copy receives a yellow frame, so as to visually differ from the original source and to remind the user that she is manipulating a copy.
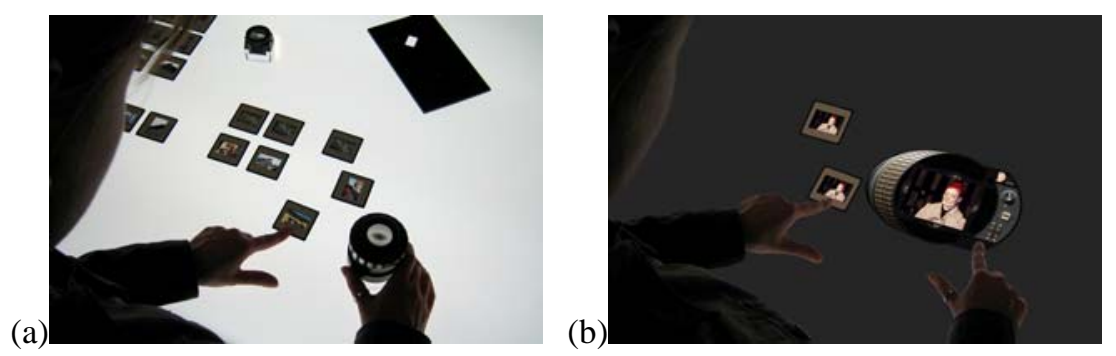

Fig. 2. Affordances for manipulation of pictures. (a) Physical slides on a light table. (b) Digital slides for the representation of image content in the EnLighTable appliance.

\subsection{Virtual Tools for Direct Manipulation of Digital Information}

Graphic designers have accustomed themselves to edit picture files with graphic desktop applications, such as Adobe Photoshop. The graphical user interface of this kind of applications usually provides different icons, representing different tools, e.g. a lasso for drawing a selection, or a bucket to fill color regions. The appearance of these icons suggests their function, but not the type of interaction. When a user, for example, selects the lasso in the toolbar and moves the pointer to the picture area, the cursor changes into a lasso. In order to draw a selection, the user is supposed to click and drag, while keeping the mouse button pressed. In contrast to this, after selecting the bucket from the toolbar and positioning the pointer on the picture, a single click will change the color of the pixels within the same color region. In terms of affordances, this means that no cognitive affordances are provided by the interface to support different mouse-based manipulation techniques.

A tighter mapping between visual representation and functionality is provided by Kai's Power Tools [5]. Based on the metaphor of a toolglass or magic lens [2], these 
Plug-Ins for Adobe Photoshop and Corel Photopaint enable the direct application and visualization of filters on the image. As a virtual tool, the lens can be dragged over different areas of the picture with the mouse, and different filter parameters can be entered with the keyboard. Moving even more towards a real direct manipulation, and beyond the WIMP paradigm of the desktop, HabilisDraw [21][3] provides a set of virtual tools. These are controlled by fingers on a DiamondTouch table-top display. In this project, tools are just a subcategory of objects: therefore objects and tools share a common manipulation vocabulary (e.g. for moving, rotating, picking up). This means that the actions that can be performed with the different tools are not necessarily coupled to visual cues suggesting how the specific tool needs to be manipulated. Rather, a manipulation vocabulary is predefined, which is the same for every object. To make an example, in HabilisDraw virtual pens are manipulated in the same way as virtual tapes, although they have different functionalities and the effect of their application has different feedbacks.

Our Imagetool is a digital virtual tool which supports basic editing of pictures. Similarly to Kai's Power Tools [5], it relies on the conceptual model of a magic lens, which in our case is controlled by two hands directly on the surface of the table. Differently from the concept of HabilisDraw, our virtual tool provides cognitive affordances for direct manipulation relying on the way we manipulate certain physical objects. In figure 3 (a), for example, the zooming gear on the left side of the tool can be "scrolled" with a continuous movement of one hand, as we would do with a physical photographic lens. Discrete interaction, such as tapping, is suggested by the 3D effect of the buttons for mirroring and saving changes, on the right side of the tool (see fig. 3 (b), labels 3 and 4). The image can be cropped by dragging the semiopaque ledgers within the lens, which resemble the blades of a four bladed easel used in darkrooms (see fig. 3 (a), and (b) label 2). Cognitive affordances are designed to suggest to the user where to position her finger for interaction. The interface supports space multiplexed input control, in the sense that different controls can be mapped to different functions, each independently accessible. We consider the different microand macro-metric specialized work of respectively dominant and non-dominant hands [12][4]. The Imagetool can be placed with one hand, likely the non-dominant one, in order to identify the frame of reference for the dominant hand. We assume that users, particularly those in our target group of designers, might use a real pen with the dominant hand for more precise interaction. Therefore, on top of the cues for interaction with fingers, we suggest points for the placement of pen-tips by yellow dots. Other basic functionalities are provided, such as rotation (see fig. 3 (b), label 5) and visualization of picture elements in 1:1 scale (see fig. 3 (b), label 6).

The edited pictures can be saved, and remain on the shared surface of the table. Designers can also draft frames for a layout (see fig. 4 (a)). They can place and resize pictures in their layout by simply dragging the pictures inside the frames. 

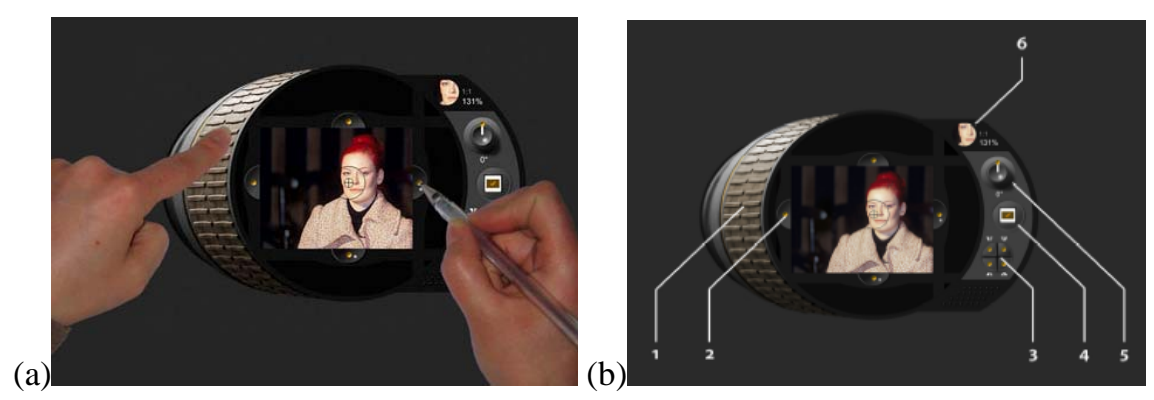

Fig. 3. (a) Bi-manual interaction with the Imagetoool. (b) Labels 1 to 5 show the interactive areas of the Imagetool. Label 6 indicates the area where the picture is displayed in 1:1 scale, and zooming factor is shown.

\section{Current Prototype and Future Work}

Our initial prototype of the EnLighTable was run on the touch-sensitive table of our lab. This table contains a NEC LCD display with a resolution of $1366 x 768$ pixels, over which a SmartTech Actalyst touch panel tracks fingers by 4 infrared cameras placed in the corners. The Prototype was implemented using Flash and the mouse driver provided by the touch panel. While this setup didn't provide full multi-handed input, it allowed us to explore our design ideas and communicate them in order to obtain feedback from potential users. This phase has been important for the specification of the design and the refinement of functional requirements, which will inform the implementation of the appliance in the instrumented room of our lab.

\subsection{Specifying Requirements for the Next Design Iteration}

So far, we adopted a qualitative approach to evaluate the functionality and the user interface of the appliance in informal trials. We conducted 7 in depth interviews with individuals who cover different roles within creative teamwork: two managers of creative agencies, two creative directors, a free lancer working on conceptual design for adverting agencies, a graphic designer, and a professor of art pedagogy. The size of the agencies they worked for was heterogeneous (up to 20 people), as well as the focus of the agencies (print communication as well as web).

Every interview lasted about 1.5 hours and followed a standard format: we first asked the interviewees some questions concerning the specific workflow of their creative teams, e.g., how many people usually work on a project, their roles and professions, how activities of picture selection take place, what activities are worked out in co-location, etc.. We then introduced them to our vision of ubiquitous displays and novel collaborative scenarios: in this context we showed them a video which illustrates how the EnLighTable works. We then lead them to the instrumented room of 
our lab and showed them the interactive table. In this phase they were first given a short demonstration of how to use the different functionalities and then they were invited to play around with it. We then engaged them in a discussion about the interface, e.g. what they found intuitive or less intuitive in the prototype; and on the potential use and impact of such an appliance in their creative teamwork.

Even though the number of tests we had so far is too limited to make generalizations, the interviews gave us some useful insights for further requirements. Furthermore they provided a better understanding of how the target medium (i.e., paper or digital communication) affects picture selection as well as organizational and collaborative aspects. The possibility to simultaneously view multiple pictures on a table, to easily drag them within the focus of attention and visual angle of team members, and to discuss them together with colleagues was highly appreciated by each interviewee. The interaction technique, which allows natural manipulation without a mouse, was also positively assessed. Three interviewees suggested that such an interaction could make the process of picture selection more accessible to everyone, potentially to customers as well, thus having customers' feedback earlier in the design process. The virtual tool was used correctly by every user and its look and feel was appreciated. Six users suggested that it would be useful to have the possibility to scribble and make annotations on the table. Two users also noted that it would be good to have standard formats (e.g., DIN A4 or letter) visualized on the grid of the digital table.

According to the specialization of the agency, further observations were made. For print agencies, the resolution of the display and the possibility to view pictures in 1:1 scale is very important. Web communication seems to require less team effort in picture selection: in this field ad-hoc shooting is quite rare, and digital libraries are mostly consulted by a single graphic designer. Furthermore, web agencies seem to have a more horizontal structure. In this case the graphic designer is often directly working with the customer, thus having less communication flow within the agency.

\subsection{The Table in the Environment}

In this paper we focused on the design of an interface for multi-user interaction on a table-top display. The peculiarities and the relatively novel features of table-top displays for digital information motivated our specific consideration of the device in isolation from other possible displays and devices in the environment. The progressive cost reduction of projectors and of large, high-resolution displays makes interactive workspaces more and more plausible in real working environments of the near future. Different collaborative tasks will need to be supported by the migration of information and activities across different displays. This will require suitable manipulation techniques and relative affordances. We plan to extend our appliance to a vertical large display in the instrumented environment of our lab (see fig. 4 (b)). Users will be able to display the designed layout on the vertical large display, so as to initiate discussion in the team. The absence of orientation conflicts and the wider area within the users' visual angle make vertical displays more suitable for the presentation activity. In our design we are addressing the ecology of displays in the environment, to meet the different needs of different phases of a collaborative workflow, and 
to enable a consistent interaction in such a display continuum. In this sense we do not expect that individual work on personal displays, supported by the respective applications for photo editing, will be replaced: rather, it will be integrated into novel collaborative scenarios.

(a)
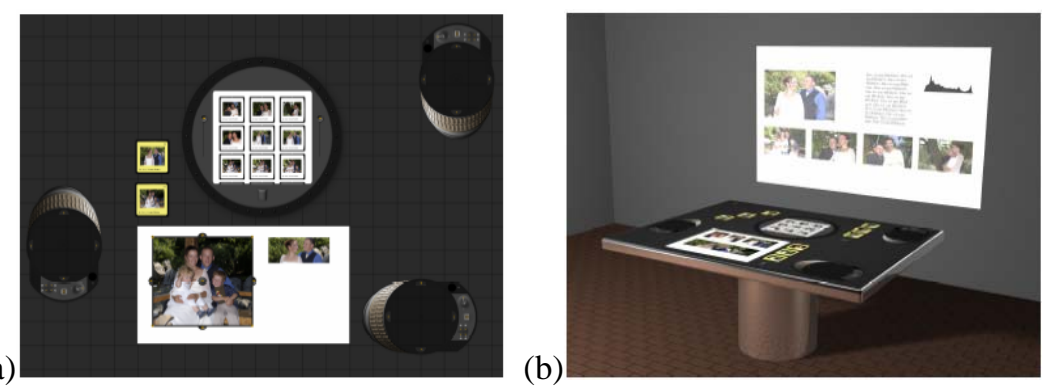

Fig. 4. Layout design on the EnLighTable: (a) In the current prototype; (b) In the extended version of the appliance, in which we plan to integrate vertical displays.

Acknowledgments. This research has been funded by Deutsche Forschungsgemeinschaft (DFG) within the FLUIDUM (Flexible User Interfaces for Distributed Ubiquitous Machinery) project. Additional Equipment was provided by the Bavarian State. We also would like to thank the people who kindly participated in our user study.

\section{References}

1. Apted, T, J Kay and A Quigley, Tabletop Sharing of Digital Photographs for the Elderly. In Proceedings of CHI2006, the Conference in Human Factors in Computing Systems, April 24 - 27, 2006, Montréal, Québec, Canada. (to appear).

2. Bier, E., Stone, M., Pier, K., Buxton, W., and DeRose, T.: Toolglasses and Magic Lenses: the Seetrough Interfaces. Proceedings of Computer Graphics, August 1993, pp73-80.

3. Butler, C. G., and St. Amant, R. (2004). HabilisDraw DT: A Bimanual Tool-Based Direct Manipulation Drawing Environment. ACM Conference on Human Factors in Computing Systems (CHI), short papers. Pp. 1301-1304.

4. Buxton, W. and Myers, B. A.: A Study in Two-Handed Input, Proc. ACM Conf on Human Factors in Computing Systems (CHI '86), 321-326.

5. Cohen, S.: Kai's Power Tools 3 for Windows Visual Quickstart Guide. Peachpit Press, 1997.

6. Dourish, P. and Bellotti, V.: Awareness and Coordination in Shared Workspaces. Proceedings of theConference on Computer Supported Cooperative Work CSCW '92, Toronto, Canada, ACM Press, New York, pp. 107-114.

7. Dourish, P. and Bly, S.: Portholes: Supporting awareness in a distributed work group. In Proc. of CHI92 : Human Factors in Computing Systems. ACM: New York. p. 541-547.

8. Dourish, P.: Where the Action is. The Foundations of Embodied Interaction. Bradfor Books, (2004).

9. Fischer, G.: Social Creativity, Symmetry of Ignorance and Meta-Design. In L. Candy and E. Edmonds (eds), Proceedings of the Conference "Creativity \& Cognition 1999", ACM Press, pp 116-123. 
10. Gaver, W.: Affordances for Interaction: the Social is Material for Design. Ecological Psychology 8 (2),

11. Grint, K., and Woolgar, S.: The Machine at Work. Polity, Cambridge, UK, 1997.

12. Guiard, Y.: Asymmetric Division of Labor in Human Skilled Bimanual Action: The Kinematic Chain as a Model. Journal Motor Behavior, 19 (4), 1987, 486-517.

13. Hartson, R., H.: Cognitive, physical, sensory, and functional affordances in interaction design. In Behavior \& Information Technology, September-October 2003, vol. 22, n. 5, 315-338.

14. Hinrichs, U., Carpendale, S., Scott, S. and Pattison E.: Interface Currents: Supporting Fluent Collaboration on Tabletop Displays. In Proceedings of the 5th Symposium on Smart Graphics, August 22-24, 2005. Frauenwörth Cloister, Germany, pp. 185-197.

15. Kirsch , D: The Intelligent Use of Space. Journal of Artificial Intelligence, 73(1-2), 31-68, 1995

16. Kreijns, K. and Kirschner, P. A.: The social affordances of computer-supported collaborative learning environments. In Proceedings of the 31th ASEE/IEEE Frontiers in Education Conference, 12-17, (2001).

17. Krüger, W., Bohn, C.A., Fröhlich, B., Schüth, H., Strauss, W., Wesche, G.: The Responsive Workbench: A Virtual Work Environment. IEEE Computer 28(7): $42-48$ (1995).

18. Rekimoto J. and Saitoh, M.: Augmented Surfaces: A Spatially Continuous Workspace for Hybrid Computing Environments. Proceedings of CHI'99, 1999.

19. Scott, S.D., Carpendale, S., \& Habelski, S.: Storage Bins: Mobile Storage for Collaborative Tabletop Displays. IEEE Computer Graphics \& Applications: Special Issue on Large Displays, 25(4), July/August 2005, pp. 58-65.

20. Scott, S.D., Carpendale, M.S.T, \& Inkpen, K.M.: Territoriality in Collaborative Tabletop Workspaces. In Proceedings of the ACM Conference on Computer-Supported Cooperative Work (CSCW)'04, November 6-10, 2004, Chicago, IL, USA.

21. St. Amant, R., and Horton, T. E. (2002).: Characterizing tool use in an interactive drawing environment. Second International Symposium on Smart Graphics. Pp. 86-93.

22. Streitz, N. A., Geißler, J. Holmer, T., Konomi, S., Müller-Tomfelde, C., Reischl, W., Rexroth, P., Seitz, P., Steinmetz R.: i-LAND: An interactive Landscape for Creativity and Innovation. In: ACM Conference on Human Factors in Computing Systems (CHI'99), Pittsburgh, Pennsylvania, USA, May 15-20, 1999. pp. 120-127.

23. Sundholm, H., Artman, H., Ramberg, R.: Backdoor Creativity - Technological support and Collaborative Creativity. In F.Darses, Zarate, P., Zackland, M. (Eds.). Proceedings of Conference on the Design of Cooperative Systems (COOP’04). Heyeres, France, May, 2004.

24. Terrenghi, L.: Design of Affordances for Direct Manipulation of Digital Information. In Proc. Smart Graphics 2005, Munich, Germany.

25. Ullmer, B. and Ishii, H., The metaDESK: Models and Prototypes for Tangible User Interfaces, in Proceedings of Symposium on User Interface Software and Technology UIST '97), (Banff, Alberta, Canada, October, 1997), ACM Press, pp. 223-232.

26. Weiser, M.: The computer for the 21st century. Scientific American, Vol. 265, September 1991.

27. Wellner. P.: Interacting with paper on the DigitalDesk. Communications of the ACM, 36(7):86--96, July 1993. 